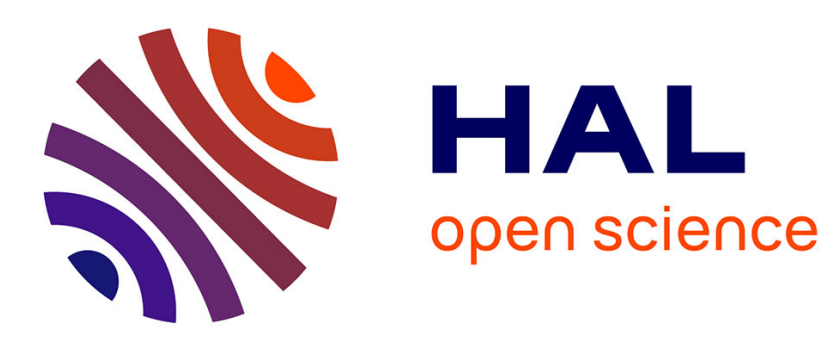

\title{
Singular perturbation analysis of a coupled system involving the wave equation
}

Eduardo Cerpa, Christophe Prieur

\section{To cite this version:}

Eduardo Cerpa, Christophe Prieur. Singular perturbation analysis of a coupled system involving the wave equation. IEEE Transactions on Automatic Control, 2020, 65 (11), pp.4846-4853. 10.1109/TAC.2019.2960272 . hal-03066813

\section{HAL Id: hal-03066813 https://hal.science/hal-03066813}

Submitted on 15 Dec 2020

HAL is a multi-disciplinary open access archive for the deposit and dissemination of scientific research documents, whether they are published or not. The documents may come from teaching and research institutions in France or abroad, or from public or private research centers.
L'archive ouverte pluridisciplinaire HAL, est destinée au dépôt et à la diffusion de documents scientifiques de niveau recherche, publiés ou non, émanant des établissements d'enseignement et de recherche français ou étrangers, des laboratoires publics ou privés. 


\title{
Singular perturbation analysis of a coupled system involving the wave equation
}

\author{
Eduardo Cerpa and Christophe Prieur
}

\begin{abstract}
This article considers a system coupling an ordinary differential equation with a wave equation through its boundary data. The existence of a small parameter in the wave equation (as a factor multiplying the time derivative) suggests the idea of applying a singular perturbation method to get the stability of the full system by analyzing the stability of some appropriate subsystems given by the method. However, for infinite-dimensional systems it is known that, in some cases, this method does not work. Indeed, one can not be sure of the stability of the full system even if the given subsystems are stable. In this paper we prove that the singular perturbation method works for the system under study. Using this strategy we get the stability of the system and a Tikhonov theorem, which is the first of this kind for systems involving the wave equation. Simulations are performed to show the applicability of our results.
\end{abstract}

\section{INTRODUCTION}

The singular perturbation method (SPM) is a classical tool to study stability properties of coupled systems where there appear some small parameters. Roughly speaking, the idea is to deduce the stability of the original system by using the behavior of the system when those parameters are chosen to be zero. Depending on the applications and the particular equations, the parameters can play the role of different time scales allowing the modeling of different physical situations. As an example we can mention the SaintVenant-Exner equations described in [13] and in [3, Section 1.5]. This hyperbolic system is used to study the dynamics of the flow in a reach, coupled with the sediment dynamics. The sediment dynamics has, by nature, a very slow dynamic with respect to the velocity flow in the fluid. Thus this model is a singularly perturbed hyperbolic system, as studied in [16] (see also [9] for control results on this system). Other examples of systems with different time scales appear when considering infinite-dimensional control systems with dynamics at the boundaries, as introduced for instance in [3, Section 3.4], and further considered in particular in [1] where sufficient conditions are derived for the stability of the coupled system. One naturally obtains partial differential equations (PDE) coupled to ordinary differential equations (ODE) at different time scales. In [22, Chapter 2] a slow

This work has been partially supported by Fondecyt 1180528, ECOS C16E06, and Basal Project FB0008 AC3E

E. Cerpa is with Departamento de Matemática, Universidad Técnica Federico Santa María, Avda. España 1680, Valparaíso, Chile. E-mail: eduardo. cerpa@usm. cl

C. Prieur is with Univ. Grenoble Alpes, CNRS, Grenoble INP, GIPSA-lab, F-38000 Grenoble, France. E-mail: christophe.prieuregipsa-lab.fr
ODE coupled with a fast PDE appears, and in [19] a fast ODE coupled with a slow PDE is studied.

As usual, the literature on singularly perturbed systems has first grown up for finite-dimensional systems (see in particular the seminal works [12], [14]). For infinite-dimensional systems, we find [10], [11] where delay systems are studied. Closer to the present contribution, let us cite [4] where a parabolic singularly perturbed PDE is considered. Regarding coupled hyperbolic PDEs, we mention [20] and [21] dealing with conservation laws and balance laws, respectively. In both papers, Lyapunov function approaches are useful to analyze stability properties.

However, the validity of the SPM in an infinitedimensional framework depends on the system. Even linear systems can show unexpected behavior. This was shown in [19] for a first order hyperbolic system with different time scales and in [5] for a second order hyperbolic system coupled to a ODE. In these papers there are examples of unstable systems for which the SPM does not work. More precisely, it natural to think that we can deduce the stability of the full system (for small parameters) when some particular subsystems (reduced and boundary layer systems) are stable. Unfortunately this natural conjecture is false. Indeed, in [19], [5] we find exemples of unstable full system giving stable subsystems. Thus, we can not deduce the stability of the full system by applying a SPM.

The main goal of this paper is to establish stability and Tikhonov results for an infinite-dimensional system by applying the SPM.

We consider as a model the wave equation coupled to an ordinary differential equation through boundary data (see [23], [8], [2] for similar couplings). More precisely, our system is given by

$$
\begin{cases}\varepsilon^{2} w_{t t}(t, x)-w_{x x}(t, x)=0, & t \geq 0,0<x<1, \\ w(t, 0)=c z(t), & t \geq 0, \\ w_{x}(t, 1)=-\varepsilon d w_{t}(t, 1), & t \geq 0, \\ \dot{z}(t)=a z(t)+b w(t, 1), & t \geq 0\end{cases}
$$

with $a, b, c, d$ constant values and a positive value $\varepsilon>0$. When $\varepsilon>0$ is small then the dynamics (1) have two different time scales and couplings, yielding to, roughly speaking, a wave equation with velocity $\frac{1}{\varepsilon}$ and an ODE with slow velocity. The sign conditions on the constant values will be justified by considering some subsystems where, in particular, it will be clear that $d>0$ and $a+b c$ are necessary and sufficient conditions for the stability of the subsystems. We consider usual initial condition for (1) given by $w^{0}$ in 
$H^{1}(0,1), w^{1}$ in $L^{2}(0,1)$ and $z^{0}$ in $\mathbb{R}$, that is

$$
\begin{cases}w(0, x)=w^{0}(x), & 0<x<1, \\ w_{t}(0, x)=w^{1}(x), & 0<x<1, \\ z(0)=z^{0} . & \end{cases}
$$

It is important to note here that the stability of this system can not be deduced from the results in [19]. Even if one-dimensional wave equations can be written in terms of first-order hyperbolic equations, system (1) presents troubles with the boundary conditions. By rewriting system (1) in Riemann coordinates, we get a system of conservation laws coupled with an ODE. However, in Riemann coordinates, the boundary conditions obtained from second and four lines in (1) are different to the ones in [19]. See Appendix A for the precise writing of system (1) in Riemann coordinates. In this way, we can see that the results in [19] do not apply for (1) and consequently our developments are a true contribution with respect to the existing literature in the topic.

When applying SPM, we have to obtain what are called the reduced and the boundary layer systems. In fact, as it will be explained in Section II below, the reduced system is

$$
\frac{d}{d t} \bar{z}=(a+b c) \bar{z}, \quad t \geq 0,
$$

while the boundary layer system for $\tau=t / \varepsilon$ is

$$
\begin{cases}\bar{w}_{\tau \tau}(\tau, x)-\bar{w}_{x x}(\tau, x)=0, & \tau \geq 0,0<x<1, \\ \bar{w}(\tau, 0)=0, & \tau \geq 0, \\ \bar{w}_{x}(\tau, 1)=-d \bar{w}_{\tau}(\tau, 1), & \tau \geq 0 .\end{cases}
$$

We consider usual initial condition for (3) and (4) given by $\bar{w}^{0}$ in $H^{1}(0,1), \bar{w}^{1}$ in $L^{2}(0,1)$ and $\bar{z}^{0}$ in $\mathbb{R}$, that is

$$
\begin{cases}\bar{w}(0, x)=\bar{w}^{0}(x), & 0<x<1, \\ \bar{w}_{t}(0, x)=\bar{w}^{1}(x), & 0<x<1\end{cases}
$$

and

$$
\bar{z}(0)=\bar{z}^{0} \text {. }
$$

Remark 1: Let us notice that the stability of the reduced system (3) is equivalent to $a+b c<0$ and the stability of the boundary layer (4) is equivalent to $d>0$ (this can be proved for instance using the Lyapunov function (11) obtained in [18]). Thus, our first result is concerned with the stability of system (1) when the subsystems are stable and $\varepsilon$ is small enough. It is worth to mention that Theorem 1 below appeared in the conference paper [5] but we give here a different proof. The used approach allows to unify the stability analysis in Theorem 1 with the Tikhonov result in Theorem 2.

Theorem 1: Let $d>0$ and $a, b, c$ such that $a+b c<0$. There exists $\varepsilon^{\star}>0$ such that for any $\varepsilon \in\left(0, \varepsilon^{\star}\right)$ the full system (1) is exponentially stable, that is, there exists $C_{0}>$ 0 such that, for all $\left(w^{0}, w^{1}, z^{0}\right)$ in $H^{1}(0,1) \times L^{2}(0,1) \times$ $\mathbb{R}$ satisfying the compatibility condition $w^{0}(0)=c z^{0}$, the solution $z \in C([0,+\infty))$ and $w \in C^{0}\left([0,+\infty) ; H^{1}(0,1)\right) \cap$ $C^{1}\left([0,+\infty) ; L^{2}(0,1)\right)$ to $(1)-(2)$ satisfies, for all $t \geq 0$,

$$
\begin{aligned}
& \left\|\left(w(t), w_{t}(t), z(t)\right)\right\|_{H^{1}(0,1) \times L^{2}(0,1) \times \mathbb{R}} \\
& \leq C_{0} e^{\frac{(a+b c)}{2} t}\left\|\left(w^{0}, w^{1}, z^{0}\right)\right\|_{H^{1}(0,1) \times L^{2}(0,1) \times \mathbb{R} .}
\end{aligned}
$$

The second result of this paper states that the SPM gives us a Tikhonov approximation: the dynamics of (1) can be approximated by those of the boundary layer system (4) and of the reduced system (3).

Theorem 2: Let $d>0$ and $a, b, c$ such that $\delta:=a+$ $\sqrt{3}|b c|<0$. There exists $\varepsilon^{\star}>0$ such that for any $\varepsilon \in\left(0, \varepsilon^{\star}\right)$, $w^{0}$ in $H^{1}(0,1), w^{1}$ in $L^{2}(0,1), z^{0}$ in $\mathbb{R}, \bar{w}^{0}$ in $H^{1}(0,1), \bar{w}^{1}$ in $L^{2}(0,1), \bar{z}^{0}$ in $\mathbb{R}$ satisfying the compatibility conditions $w^{0}(0)=c z^{0}, \bar{w}^{0}(0)=0$, and smallness conditions

$$
\begin{array}{r}
\left\|w^{0}-c \bar{z}^{0}-\bar{w}^{0}\right\|_{H^{1}(0,1)}+\left\|w^{1}-(a+b c) c \bar{z}^{0}-\bar{w}^{1}\right\|_{L^{2}(0,1)} \\
+\left|z^{0}-\bar{z}^{0}\right|=O\left(\varepsilon^{2}\right) \\
\left\|\bar{w}^{0}\right\|_{H^{1}(0,1)}+\left\|\bar{w}^{1}\right\|_{L^{2}(0,1)}=O\left(\varepsilon^{3 / 2}\right), \quad\left|\bar{z}^{0}\right|=O\left(\varepsilon^{3 / 2}\right),
\end{array}
$$

the solution $w$ in $C\left([0, \infty) ; H^{1}(0,1)\right) \cap C^{1}\left([0, \infty) ; L^{2}(0,1)\right)$ and $z$ in $C^{1}([0, \infty))$ to (1)-(2) satisfies, for all $t \geq 0$,

$$
\begin{aligned}
& \|w(t)-c \bar{z}(t)-\bar{w}(t / \varepsilon)\|_{H^{1}(0,1)} \\
& +\left\|w_{t}(t)-c(a+b c) \bar{z}(t)-\bar{w}_{\tau}(t / \varepsilon)\right\|_{L^{2}(0,1)}=e^{\delta t} O(\varepsilon),
\end{aligned}
$$

and

$$
|z(t)-\bar{z}(t)|=e^{\delta t} O\left(\varepsilon^{3 / 2}\right),
$$

where $\bar{w}$ in $C\left([0, \infty) ; H^{1}(0,1)\right) \cap C^{1}\left([0, \infty) ; L^{2}(0,1)\right)$ is the solution to (4) and (5) and $\bar{z}$ in $C^{1}([0, \infty))$ is the solution to (3) and (6).

Note that the assumptions on coefficients $a, b, c$ are more restrictive in Theorem 2 than in Theorem 1 since $\delta>a+b c$. Thus, the exponential decay rate in Theorem 2 is weaker than the one in Theorem 1. The interest of Theorem 2 is to give first order and second order approximations of the full system using the subsystems. Roughly speaking, it does not only imply that system goes to zero, but it explains how it does when the parameter $\varepsilon$ is small enough. Moreover, we note that regarding the solution $w$ there is a $O\left(\varepsilon^{2}\right)$ in the initial condition hypothesis and a $O(\varepsilon)$ in the conclusions of Theorem 2. This is due to the fact that our Lyapunov function depends on $\varepsilon$. Since our method is based on the derivation of a strict Lyapunov function, we get also a robustness with respect to some parameters, as the speed of the wave and the estimation of the errors with the boundary layer and reduced systems.See the proof of Theorem 2 for more details. Only scalar wave and ODE equations are considered in this paper. The generalization to vectorial cases is omitted to ease the reading of this paper.

The remaining part of the paper is organized as follows. In Section II we prove Theorem 1. Section III is devoted to the proof of Theorem 2. Section IV contains numerical simulations illustrating the stability and the Tikhonov approximation stated in our theorems. Finally, we give in Section V some conclusions. Appendix A shows as our system is written in Riemann coordinates while Appendix $\mathrm{B}$ is concerned with an important technical lemma.

Notation: In this section and in the remaining part of this paper, $|\cdot|$ is the absolute value. Given any function 
$u=u(t, x), u_{t}$ and $u_{x}$ denote the partial derivatives with respect to $t$ and $x$, respectively. The set $L^{2}(0,1)$ is the set of measurable functions $v:[0,1] \mapsto \mathbb{R}$ such that $\|v\|_{L^{2}(0,1)}:=\sqrt{\int_{0}^{1} v(x)^{2} d x}<\infty$. The set $H^{1}(0,1)$ is the set of functions in $L^{2}(0,1)$ such that $\|v\|_{H^{1}(0,1)}:=$ $\sqrt{\|v\|_{L^{2}(0,1)}^{2}+\left\|v_{x}\right\|_{L^{2}(0,1)}^{2}}<\infty$. If $v \in H^{1}(0,1)$ and additionally $v(0)=0$ and/or $v(1)=0$, then we can use the equivalent norm $\sqrt{\left\|v_{x}\right\|_{L^{2}(0,1)}^{2}}$ thanks to Poincaré inequality.

\section{Stability ANALYSis}

The goal of this section is to prove Theorem 1. We apply the SPM, which leads us to find some appropriate subsystems called the reduced order system and the boundary layer system. Theorem 1 makes sure that the full system is stable when the previous subsystems are stable and the parameter $\varepsilon$ is small enough.

To formally compute the reduced order system, let $\varepsilon=0$ in (1). We get from the boundary condition at $x=1$ that $w_{x}(t, 1)=0$ which gives $w_{x}=0$ when using $w_{x x}=0$ (coming from the PDE). From the boundary condition at $x=0$, it follows that $w(t, x)=c z(t)$ for all $t \geq 0$ and for all $x \in(0,1)$. Thus, the reduced order system is

$$
\frac{d}{d t} \bar{z}=(a+b c) \bar{z}, \quad t \geq 0 .
$$

Let us compute now the boundary layer system. We introduce $\tau=t / \varepsilon$ and let us define formally the variable $\bar{w}(\tau, x)=w(\tau, x)-c z(\tau)$ when $\varepsilon=0$. We compute $\frac{d}{d \tau} \bar{w}(\tau, x)=\frac{d}{d \tau} w(\tau, x)-c \varepsilon \frac{d}{d t} z(\tau)=\frac{d}{d \tau} w(\tau, x)-$ $c \varepsilon(a z(\tau)+b w(\tau, 1))$, by using the $z$ dynamics. Therefore, letting $\varepsilon=0$, we formally get $\frac{d}{d \tau} \bar{w}=\frac{d}{d \tau} w$. Moreover, $\frac{d^{2}}{d \tau^{2}} \bar{w}=\frac{d^{2}}{d \tau^{2}} w, \frac{d}{d x} \bar{w}=\frac{d}{d x} w$, and $\frac{d^{2}}{d x^{2}} \bar{w}=\frac{d^{2}}{d x^{2}} w$. Therefore $\bar{w}_{\tau \tau}-\bar{w}_{x x}=0$. To compute the boundary conditions for the variable $\bar{w}$, let us note that $\bar{w}_{x}(\tau, 1)=w_{x}(\tau, 1)=$ $-d \varepsilon w_{t}(\tau, 1)=-d w_{\tau}(\tau, 1)=-d \bar{w}_{\tau}(\tau, 1)$ by approximating $\varepsilon$ by 0 in the last equation. To sum up, the boundary layer system is written as

$$
\begin{cases}\bar{w}_{\tau \tau}(\tau, x)-\bar{w}_{x x}(\tau, x)=0, & \tau \geq 0,0<x<1, \\ \bar{w}(\tau, 0)=0, & \tau \geq 0, \\ \bar{w}_{x}(\tau, 1)=-d \bar{w}_{\tau}(\tau, 1), & \tau \geq 0,\end{cases}
$$

defining $\bar{w}$, up to an initial condition. The boundary layer system is known to be exponentially stable. In fact, this system is called a passive damped wave equation. As the following computation is used a couple of times later, we explain it here. Let us consider the following Lyapunov function

$$
V_{1}(\bar{w})=\int_{0}^{1} e^{\mu x}\left(\bar{w}_{x}+\bar{w}_{\tau}\right)^{2} d x+\int_{0}^{1} e^{-\mu x}\left(\bar{w}_{x}-\bar{w}_{\tau}\right)^{2} d x,
$$

with $\mu>0$ to be fixed later. This Lyapunov function appeared for the wave equation in [18] and is related to a Lyapunov function for first-order hyperbolic equations studied in [7].
Along the solutions to (10), it holds

$$
\begin{aligned}
\frac{d}{d \tau} V_{1}= & 2 \int_{0}^{1} e^{\mu x}\left(\bar{w}_{\tau}+\bar{w}_{x}\right)\left(\bar{w}_{\tau \tau}+\bar{w}_{x \tau}\right) d x \\
& +2 \int_{0}^{1} e^{-\mu x}\left(\bar{w}_{\tau}-\bar{w}_{x}\right)\left(\bar{w}_{\tau \tau}-\bar{w}_{x \tau}\right) d x, \\
= & 2 \int_{0}^{1} e^{\mu x}\left(\bar{w}_{\tau}+\bar{w}_{x}\right)\left(\bar{w}_{x x}+\bar{w}_{x \tau}\right) d x \\
& -2 \int_{0}^{1} e^{-\mu x}\left(\bar{w}_{\tau}-\bar{w}_{x}\right)\left(\bar{w}_{x \tau}-\bar{w}_{x x}\right) d x, \\
= & -\mu \int_{0}^{1} e^{\mu x}\left(\bar{w}_{\tau}+\bar{w}_{x}\right)^{2} d x \\
& +\left[e^{\mu x}\left(\bar{w}_{\tau}+\bar{w}_{x}\right)^{2}\right]_{x=1}^{x=1} \\
& -\mu \int_{0}^{1} e^{-\mu x}\left(\bar{w}_{\tau}-\bar{w}_{x}\right)^{2} d x \\
& -\left[e^{-\mu x}\left(\bar{w}_{\tau}-\bar{w}_{x}\right)^{2}\right]_{x=1}^{x=1} .
\end{aligned}
$$

Now, note that the boundary condition in the second line of (10) implies that $\bar{w}_{\tau}(\tau, 0)=0$ and thus, for all $\tau \geq 0$,

$$
\begin{array}{r}
{\left[e^{\mu x}\left(\bar{w}_{\tau}+\bar{w}_{x}\right)^{2}\right](\tau, 0)-\left[e^{-\mu x}\left(\bar{w}_{\tau}-\bar{w}_{x}\right)^{2}\right](\tau, 0)} \\
=\bar{w}_{x}^{2}(\tau, 0)-\bar{w}_{x}^{2}(\tau, 0)=0 .
\end{array}
$$

Therefore, we get

$$
\begin{aligned}
\frac{d}{d \tau} V_{1}= & -\mu V_{1}+e^{\mu}\left(\bar{w}_{\tau}(\tau, 1)+\bar{w}_{x}(\tau, 1)\right)^{2} \\
& -e^{-\mu}\left(\bar{w}_{\tau}(\tau, 1)-\bar{w}_{x}(\tau, 1)\right)^{2}
\end{aligned}
$$

and thus with the boundary condition in the last line of (10):

$$
\begin{aligned}
\frac{d}{d \tau} V_{1}= & -\mu V_{1}+e^{\mu}\left(\bar{w}_{\tau}(\tau, 1)-d \bar{w}_{\tau}(\tau, 1)\right)^{2} \\
& -e^{-\mu}\left(\bar{w}_{\tau}(\tau, 1)+d \bar{w}_{\tau}(\tau, 1)\right)^{2} \\
= & -\mu V_{1} \\
& +\left(e^{\mu}(1-d)^{2}-e^{-\mu}(1+d)^{2}\right) \bar{w}_{\tau}(\tau, 1)^{2} .
\end{aligned}
$$

We obtain the exponential stability by choosing $\mu$ such that $e^{\mu}(1-d)^{2}<e^{-\mu}(1+d)^{2}$, which is possible due to $d>0$.

Let us now define the following variable $\tilde{w}(t, x)=$ $w(t, x)-c z(t)$ (notice that the previously defined $\bar{w}$ is an approximation of $\tilde{w}$ for small $\varepsilon$ ). We compute successively

$$
\begin{aligned}
w_{t}= & \tilde{w}_{t}+(a+b c) c z+b c \tilde{w}(t, 1), \\
w_{t t}= & \tilde{w}_{t t}+\left(a b c+b^{2} c^{2}\right) \tilde{w}(t, 1) \\
& +b c \tilde{w}_{t}(t, 1)+\left(a^{2} c+2 a b c^{2}+b^{2} c^{3}\right) z, \\
\tilde{w}_{x}= & w_{x}, \\
\tilde{w}_{x x}= & w_{x x} .
\end{aligned}
$$

Therefore we get the following dynamics, equivalent to (1)

$$
\left\{\begin{array}{l}
\varepsilon^{2} \tilde{w}_{t t}-\tilde{w}_{x x}+\varepsilon^{2}\left(a b c+b^{2} c^{2}\right) \tilde{w}(t, 1) \\
\quad+\varepsilon^{2} b c \tilde{w}_{t}(t, 1)+\varepsilon^{2}\left(a^{2} c+2 a b c^{2}+b^{2} c^{3}\right) z(t)=0, \\
\tilde{w}(t, 0)=0 \\
\tilde{w}_{x}(t, 1)=-\varepsilon b c d \tilde{w}(t, 1)-d \varepsilon \tilde{w}_{t}(t, 1)-\varepsilon d(a+b c) c z(t), \\
\dot{z}(t)=(a+b c) z(t)+b \tilde{w}(t, 1) .
\end{array}\right.
$$

We are now in position to prove Theorem 1 by studying system (13).

Proof: In order to prove that system (13) is exponentially stable, we apply Lemma 1 in Appendix B. More precisely, we use (23) with $A=-\left(a^{2} c+2 a b c^{2}+b^{2} c^{3}\right)$, $B=-\left(a b c+b^{2} c^{2}\right), C=-b c, D=-d, E=-d(a+b c) c$, $F=-b c d, G=a+b c, H=b, M=0, d_{1}(t)=$ $d_{2}(t)=d_{3}(t)=0$, for all $t \geq 0$. Thus, defining $V(\tilde{w}, z)=$ $V_{1}(\tilde{w})+V_{2}(z)$, with

$$
V_{1}=\int_{0}^{1} e^{\mu x}\left(\tilde{w}_{x}+\varepsilon \tilde{w}_{t}\right)^{2} d x+\int_{0}^{1} e^{-\mu x}\left(\tilde{w}_{x}-\varepsilon \tilde{w}_{t}\right)^{2} d x
$$


and $V_{2}=z^{2}$, we get that, along the solutions to (13),

$$
\begin{gathered}
\frac{d}{d t} V \leq\left[-\frac{\mu}{\varepsilon}+e^{\mu}\left(\kappa_{1}+\kappa_{2}+\kappa_{3}+\kappa_{4}\right)+\frac{2 \varepsilon^{2} B^{2} C_{1}}{\kappa_{2}}\right. \\
\left.+3 \varepsilon C_{1} F^{2}\left(e^{\mu}+e^{-\mu}\right)\left(1+\frac{|D|+1}{\kappa_{5}}\right)+\frac{C_{1} H^{2}}{\kappa_{6}}\right] V_{1} \\
+\left[2 G+\kappa_{6}+\kappa_{7}+\frac{2 \varepsilon^{2} A^{2}}{\kappa_{1}}+3 \varepsilon E^{2}\left(e^{\mu}+e^{-\mu}\right)\left(1+\frac{|D|+1}{\kappa_{5}}\right)\right] V_{2} \\
+\left[\varepsilon e^{\mu}(D+1)^{2}-\varepsilon e^{-\mu}(D-1)^{2}+3 \varepsilon \kappa_{5}\left(e^{\mu}+e^{-\mu}\right)(|D|+1)\right. \\
\left.+\frac{2 \varepsilon^{2} C^{2}}{\kappa_{3}}\right] \beta_{t}(t, 1)^{2}
\end{gathered}
$$

for all positive values $\kappa_{i}$ with $i \in\{1,2, \ldots, 7\}$.

Note that, under the assumptions of Theorem $1, D<0$ and consequently $-(D-1)^{2}+(D+1)^{2}<0$. Now letting

$$
\kappa_{5}=\frac{1}{12} \frac{\left|(D+1)^{2}-(D-1)^{2}\right|}{(|D|+1)}
$$

we get the existence of $\mu^{\star}$ such that, for all $\mu$ in $\left(0, \mu^{\star}\right]$,

$e^{\mu}(D+1)^{2}-e^{-\mu}(D-1)^{2}+3 \kappa_{5}\left(e^{\mu}+e^{-\mu}\right)(|D|+1)<0$.

Then for any positive $\kappa_{3}$ we get the existence of $\varepsilon^{\star}$ such that, for all $\varepsilon$ in $\left(0, \varepsilon^{\star}\right)$,

$$
\begin{array}{r}
\varepsilon e^{\mu}(D+1)^{2}-\varepsilon e^{-\mu}(D-1)^{2}+3 \varepsilon \kappa_{5}\left(e^{\mu}+e^{-\mu}\right)(|D|+1) \\
+\frac{2 \varepsilon^{2} C^{2}}{\kappa_{3}}<0 .
\end{array}
$$

In this way, we obtain, along the solutions to (13),

$$
\begin{gathered}
\frac{d}{d t} V \leq\left[-\frac{\mu}{\varepsilon}+e^{\mu}\left(\kappa_{1}+\kappa_{2}+\kappa_{3}+\kappa_{4}\right)+\frac{2 \varepsilon^{2} B^{2} C_{1}}{\kappa_{2}}\right. \\
\left.+3 \varepsilon C_{1} F^{2}\left(e^{\mu}+e^{-\mu}\right)\left(1+\frac{|D|+1}{\kappa_{5}}\right)+\frac{C_{1} H^{2}}{\kappa_{6}}\right] V_{1} \\
+\left[2 G+\kappa_{6}+\kappa_{7}+\frac{2 \varepsilon^{2} A^{2}}{\kappa_{1}}\right. \\
\left.+3 \varepsilon E^{2}\left(e^{\mu}+e^{-\mu}\right)\left(1+\frac{|D|+1}{\kappa_{5}}\right)\right] V_{2} .
\end{gathered}
$$

We make negative the factor multiplying $V_{2}$ in (15). As $G<0, A=O(1), D=O(1), E=O(1)$, for any positive value $\kappa_{1}$, there exist sufficiently small values $\kappa_{6}>0, \kappa_{7}>0$ and $\varepsilon^{\star}$, such that for all $\varepsilon$ in $\left(0, \varepsilon^{\star}\right)$ (up to reducing $\varepsilon^{\star}$ ) and for all $\mu$ in $\left(0, \mu^{\star}\right)$, it holds

$2 G+\kappa_{6}+\kappa_{7}+\frac{2 \varepsilon^{2} A^{2}}{\kappa_{1}}+3 \varepsilon E^{2}\left(e^{\mu}+e^{-\mu}\right)\left(1+\frac{|D|+1}{\kappa_{5}}\right)<\frac{3}{2} G$.

Remark 2: Of course, in previous line the factor $\frac{3}{2}$ multiplying $G$ can be changed to any factor in the interval $(0,2)$. We can get a factor as near to 2 as we want.

Thanks to the term $-\mu / \varepsilon$ we can make negative the factor multiplying $V_{1}$ in (15). As $B=O(1), D=O(1), F=O(1)$, and $H=O(1)$, for any positive value $\kappa_{2}>0$ we have that for all $\varepsilon$ in $\left(0, \varepsilon^{\star}\right)$ (up to reducing $\left.\varepsilon^{\star}\right)$ and for all $\mu$ in $\left(0, \mu^{\star}\right)$, it holds

$$
\begin{aligned}
& -\frac{\mu}{\varepsilon}+e^{\mu}\left(\kappa_{1}+\kappa_{2}+\kappa_{3}+\kappa_{4}\right)+\frac{2 \varepsilon^{2} B^{2} C_{1}}{\kappa_{2}} \\
& +3 \varepsilon C_{1} F^{2}\left(e^{\mu}+e^{-\mu}\right)\left(1+\frac{|D|+1}{\kappa_{5}}\right)+\frac{C_{1} H^{2}}{\kappa_{6}}<-\frac{\mu}{2 \varepsilon} .
\end{aligned}
$$

We arrive in this way to

$$
\begin{aligned}
\frac{d}{d t} V(t) \leq-\frac{\mu}{2 \varepsilon} V_{1}(t)+\frac{3}{2} G V_{2}(t) \leq-\min & \left\{\frac{\mu}{2 \varepsilon}, \frac{3}{2} G\right\} V(t) \\
& \leq-\frac{3}{2} G V(t),
\end{aligned}
$$

for all $\varepsilon \in\left(0, \varepsilon^{\star}\right)$ with a sufficiently small positive value $\varepsilon^{\star}$.

Therefore, the function $V$ decreases to zero exponentially fast, along the solutions to (13). Note that this exponential decreasing for the Lyapunov function $V(\tilde{w}, z)$ is equivalent, up to a factor $\varepsilon^{2}$, to the exponential decreasing of the usual norm in $H^{1}(0,1) \times L^{2}(0,1) \times \mathbb{R}$ thanks to the fact that $\tilde{w}(t, 0)=0$.

This concludes the proof of Theorem 1.

\section{TIKHONOV THEOREM}

Until now we have seen that the SPM gives us the reduced order system and the boundary layer, whose stability imply the stability of the full system. The goal of this section is to prove Theorem 2, that uses the subsystems to give a better approximation of the full system. The idea is not only saying that the system goes to zero but explaining how it does when the parameter $\varepsilon$ is small enough. To be more specific we introduce the errors between the solutions to (1)-(2) with the solutions to the boundary layer system (4) and (5) and to the reduced system (3) and (6). This justify the introduction of the following variables, for all $t \geq 0$ and $x$ in $[0,1]$,

$$
\alpha(t)=z(t)-\bar{z}(t)
$$

and

$$
\beta(t, x)=w(t, x)-c \bar{z}(t)-\bar{w}\left(\frac{t}{\varepsilon}, x\right) .
$$

From (1) and (9), we get

$$
\begin{aligned}
\dot{\alpha}(t) & =a z(t)+b w(t, 1)-(a+b c) \bar{z}(t) \\
& =a \alpha(t)-b c \bar{z}(t)+b w(t, 1) \\
& =a \alpha(t)+b \beta(t, 1)+b \bar{w}\left(\frac{t}{\varepsilon}, 1\right) .
\end{aligned}
$$

Moreover, we compute successively

$$
\begin{gathered}
\beta_{t}(t, x)=w_{t}(t, x)-c(a+b c) \bar{z}-\frac{1}{\varepsilon} \bar{w}_{\tau}\left(\frac{t}{\varepsilon}, x\right), \\
\beta_{t t}(t, x)=w_{t t}(t, x)-c(a+b c)^{2} \bar{z}-\frac{1}{\varepsilon^{2}} \bar{w}_{\tau \tau}\left(\frac{t}{\varepsilon}, x\right), \\
\beta_{x}(t, x)=w_{x}(t, x)-\bar{w}_{x}\left(\frac{t}{\varepsilon}, x\right),
\end{gathered}
$$

and

$$
\beta_{x x}(t, x)=w_{x x}(t, x)-\bar{w}_{x x}\left(\frac{t}{\varepsilon}, x\right) .
$$

Therefore, from (1) and (10) we get

$$
\left\{\begin{array}{l}
\varepsilon^{2} \beta_{t t}-\beta_{x x}=-\varepsilon^{2} c(a+b c)^{2} \bar{z}, \\
\beta(t, 0)=c(z(t)-\bar{z}(t)), \\
\beta_{x}(t, 1)=-d \varepsilon w_{t}(t, 1)+d \bar{w}_{\tau}\left(\frac{t}{\varepsilon}, 1\right) .
\end{array}\right.
$$


The last boundary condition of (17) is

$$
\beta_{x}(t, 1)=-d \varepsilon \beta_{t}(t, 1)-d \varepsilon c(a+b c) \bar{z}(t)
$$

where the expression of $\beta_{t}$ has been used. To sum up the dynamics of $\alpha$ and $\beta$ can be rewritten as

$$
\left\{\begin{aligned}
\varepsilon^{2} \beta_{t t}-\beta_{x x} & =-\varepsilon^{2} c(a+b c)^{2} \bar{z}(t) \\
\beta(t, 0) & =c \alpha(t) \\
\beta_{x}(t, 1) & =-d \varepsilon \beta_{t}(t, 1)-d \varepsilon c(a+b c) \bar{z}(t) \\
\dot{\alpha}(t) & =a \alpha(t)+b \beta(t, 1)+b \bar{w}\left(\frac{t}{\varepsilon}, 1\right)
\end{aligned}\right.
$$

We are now in position to prove Theorem 2 by studying stability of system (18).

Proof: Let us apply Lemma 1 with $A=B=C=$ $E=F=0, D=-d, G=a, H=b$, and $M=c$. For now we keep the functions $d_{1}(t), d_{2}(t)$, and $d_{3}(t)$. With $V$ defined as $V(\beta, \alpha)=V_{1}(\beta)+V_{2}(\alpha)$ with

$$
V_{1}(\beta)=\int_{0}^{1} e^{\mu x}\left(\beta_{x}+\varepsilon \beta_{t}\right)^{2} d x+\int_{0}^{1} e^{-\mu x}\left(\beta_{x}-\varepsilon \beta_{t}\right)^{2} d x
$$

and $V_{2}(\alpha)=\alpha^{2}$, we get (24) along the solutions to (18). Thus,

$$
\begin{aligned}
\frac{d}{d t} V(\beta, \alpha) \leq & {\left[-\frac{\mu}{\varepsilon}+e^{\mu}\left(\kappa_{1}+\kappa_{2}+\kappa_{3}+\kappa_{4}\right)\right.} \\
& \left.+6 c^{2} C_{1} \varepsilon b^{2}+\frac{C_{1} b^{2}}{\kappa_{6}}\right] V_{1}(\beta) \\
+\left[2 a+\kappa_{6}+\right. & \left.\kappa_{7}+3 c^{2} \frac{b^{2}}{\kappa_{6}}+6 c^{2} \varepsilon C_{1} a^{2}+18 c^{4} \varepsilon b^{2}\right] V_{2}(\alpha) \\
+ & \frac{2 \varepsilon^{2}}{\kappa_{4}} d_{1}(t)^{2}+\left(6 c^{2} \varepsilon+\frac{1}{\kappa_{7}}\right) d_{3}(t)^{2} \\
+ & 3 \varepsilon\left(e^{\mu}+e^{-\mu}\right)\left(1+\frac{|d|+1}{\kappa_{5}}\right) d_{2}(t)^{2} \\
+\left[\varepsilon e^{\mu}(1-d)^{2}-\right. & \left.\varepsilon e^{-\mu}(1+d)^{2}+3 \varepsilon \kappa_{5}\left(e^{\mu}+e^{-\mu}\right)(|d|+1)\right] \beta_{t}(t, 1)^{2} .
\end{aligned}
$$

We select $\kappa_{i}, i=1, \ldots, 5$ and $\kappa_{7}$ by adapting the proof of Theorem 1. See in particular from (14) to (16). In this way we get

$$
\begin{aligned}
\frac{d}{d t} V \leq & \left(2 a+\kappa_{6}+3 c^{2} \frac{b^{2}}{\kappa_{6}}\right) V+\frac{2 \varepsilon^{2}}{\kappa_{4}} d_{1}(t)^{2} \\
& +3 \varepsilon\left(e^{\mu}+e^{-\mu}\right)\left(1+\frac{|d|+1}{\kappa_{5}}\right) d_{2}(t)^{2} \\
& +\left(6 c^{2} \varepsilon+\frac{1}{\kappa_{7}}\right) d_{3}(t)^{2} \\
\leq \quad & (2 a+2 \sqrt{3}|c b|) V+\frac{2 \varepsilon^{2}}{\kappa_{4}} d_{1}(t)^{2} \\
& +3 \varepsilon\left(e^{\mu}+e^{-\mu}\right)\left(1+\frac{|d|+1}{\kappa_{5}}\right) d_{2}(t)^{2} \\
& +\left(6 c^{2} \varepsilon+\frac{1}{\kappa_{7}}\right) d_{3}(t)^{2}
\end{aligned}
$$

with $\kappa_{6}=\sqrt{3}|c b|\left(\right.$ minimizing $\left.\kappa_{6}+3 c^{2} \frac{b^{2}}{\kappa_{6}}\right)$. Now we use the expressions

$$
\begin{aligned}
& d_{1}(t)=-c(a+b c)^{2} \bar{z}(t), \\
& d_{2}(t)=-c d(a+b c) \bar{z}(t),
\end{aligned}
$$

and

$$
d_{3}(t)=b \bar{w}\left(\frac{t}{\varepsilon}, 1\right)
$$

Due to (9), it holds $|\bar{z}(t)| \leq e^{(a+b c) t}|\bar{z}(0)|$ and we note that there exists a constant value $C_{2}>0$ such that

$$
d_{1}(t)^{2}+d_{2}(t)^{2} \leq C_{2} e^{2(a+b c) t} \bar{z}(0)^{2} .
$$

Let us remark that under the hypothesis $a+\sqrt{3}|b c|<0$, we also have $a+b c<0$, which is essential when dealing with $\bar{z}$. Concerning $d_{3}(t)$, from (12) we can easily see that there exists $C_{3}>0$ such that, for all $t \geq 0$

$$
d_{3}(t)^{2} \leq C_{3} e^{-\frac{\mu t}{\varepsilon}}\left(\left\|\bar{w}^{0}\right\|_{H^{1}(0,1)}+\left\|\bar{w}^{1}\right\|_{L^{2}(0,1)}\right)^{2} .
$$

Inspecting the choices of $\kappa_{4}$ and $\kappa_{5}$ done in (16) and (14) respectively, we have that $\kappa_{4}=O(1), \kappa_{5}=O(1)$ and $\kappa_{7}=$ $O(1)$. Note that $2 \delta=2 a+2 \sqrt{3}|b c|$ where $\delta$ is negative and defined in the statement of Theorem 2. We first bound (19) using (20)-(21) to get

$$
\begin{gathered}
\frac{d}{d t} V \leq 2 \delta V+O\left(\varepsilon^{2}\right) e^{2(a+b c) t} \bar{z}(0)^{2}+O(\varepsilon) e^{2(a+b c) t} \bar{z}(0)^{2} \\
+O(1) e^{-\frac{\mu t}{\varepsilon}}\left(\left\|\bar{w}^{0}\right\|_{H^{1}(0,1)}+\left\|\bar{w}^{1}\right\|_{L^{2}(0,1)}\right)^{2}
\end{gathered}
$$

and then we integrate between 0 and $t$ to obtain (with $\delta>G$ )

$$
\begin{aligned}
V & \leq e^{2 \delta t}\left(V(0)+\bar{z}(0)^{2}+\left(\left\|\bar{w}^{0}\right\|_{H^{1}(0,1)}+\left\|\bar{w}^{1}\right\|_{L^{2}(0,1)}\right)^{2}\right) \\
& \leq e^{2 \delta t} O\left(\varepsilon^{3}\right)
\end{aligned}
$$

where we used the hypothesis on the initial conditions. We have now to come back to the norm. To do that we use that there exists a positive constant $C_{4}$, not depending on $\varepsilon$, such that

$$
\begin{aligned}
& \varepsilon^{2} C_{4}\left(\|f\|_{H^{1}(0,1)}+\|g\|_{L^{2}(0,1)}\right)^{2} \\
& \quad \leq \int_{0}^{1} e^{\mu x}\left(f_{x}+\varepsilon g\right)^{2} d x+\int_{0}^{1} e^{-\mu x}\left(f_{x}-\varepsilon g\right)^{2} d x .
\end{aligned}
$$

From here we deduce Theorem 2.

\section{NUMERICAL SIMULATIONS}

In this section, we illustrate Theorems 1 and 2, by some numerical simulations. We apply a Lax-Friedrichs method [17] to get the numerical solutions. The codes are available on [6].

Concerning Theorem 1, we simulate system (1) with $a=$ $-2, b=1, c=-2$, and $d=0.5$ and the initial conditions $w^{0}(x)=2 \pi \sin (2 \pi x), w^{1}(x)=2$, for all $x$ in $(0,1)$ and $z^{0}=w^{0}(1) / c$, so that the assumptions of Theorem 1 hold. Pick $\varepsilon=0.1$ for the time scale. We can check on Figure 1 that the Lyapunov function $V$ (with $\mu=0$ ) in the proof of Theorem 1 decreases exponentially fast to zero. We also see that the norm of the solution goes to zero. This is consistent with the conclusions of Theorem 1 giving the exponential stability of the full coupled system.

To illustrate Theorem 2, we also compute the numerical solutions to the reduced system (3), and to the boundary layer system (4) with the initial conditions $\bar{z}^{0}=z^{0}, \bar{w}^{0}=$ $w^{0}-c \bar{z}^{0}$, and $\bar{w}^{1}(x)=w^{1}-c(a+b c) \bar{z}^{0}$, for all $x$ in $(0,1)$. We show the norms of the Tikhonov approximations 
as given in (7). We compare a first approximation of $w$ given by the reduced system $c \bar{z}(t)$ with a second approximation given by the sum of the reduced system and the boundary layer $c \bar{z}(t)+\bar{w}(t / \varepsilon, x))$. We check on Figure 2 that the second approximation is better than the first one, specially for small times, which is natural because the contribution of the boundary layer $\bar{w}(t / \varepsilon, x)$ is relevant for small times. Thus, the interest of the Tikhonov approximation is confirmed as stated in Theorem 2.

Using the code [6], we can numerically estimate the optimal values of $\varepsilon^{\star}$ in Theorem 1 and Theorem 2 for this example. It is obtained $\varepsilon^{\star} \sim 0.42$ and $\varepsilon^{\star} \sim 0.13$, respectively.

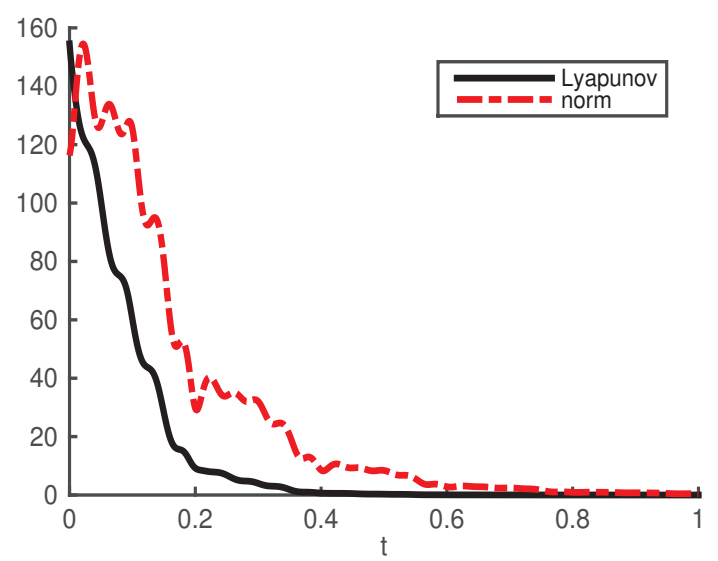

Fig. 1. Time evolution of the Lyapunov function $V$ and the norm along the solution to (1)

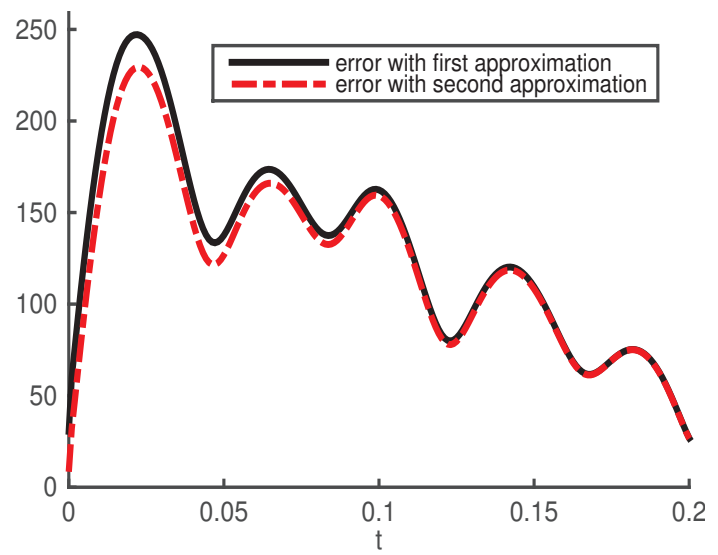

Fig. 2. Errors between the solution to (1) with the first approximation (solid back line) and with the second approximation (dashed red line)

\section{Conclusions}

In this paper we have considered an infinite-dimensional system coupling a wave equation with a linear ODE. Some small parameters appear in the partial differential equation, which can be interpreted as having different time scales. Having in mind what is known for finite-dimensional systems, it is natural to apply the singular perturbation method. However, this does not always work in an infinite-dimensional framework as shown in recent literature (see the counterexample in [20]). In this context, we prove here that this kind of analysis can be performed for the system under study and we obtain stability and Tikhonov results. Our main tool is the use of Lyapunov functions. Simulations illustrating our results are presented.

Interesting open problems arise. For instance, performing similar analysis for other infinite-dimensional systems, maybe involving nonlinearities. Other possible research line is to relax the assumptions on the initial conditions in Theorem 2 with respect to their dependence on $\varepsilon$. This would ask for looking for a Lyapunov function not depending on $\varepsilon$. Another possible research line could be to consider more than two time scales in the dynamics. Finally connections of the present work with neutral equations may be an interesting open question.

\section{APPENDIX}

\section{A. Rewriting system (1) in Riemann coordinates}

Denote $v_{1}(t, x)=\varepsilon w_{t}(t, x)+w_{x}(t, x), v_{2}(t, x)=$ $w_{x}(t, x)-\varepsilon w_{t}(t, x)$, and $Z(t)=\dot{z}(t)$. By differentiating the second and the fourth line of (1), we obtain from (1) the following system, for all $t \geq 0$, and for all $0<x<1$,

$$
\left\{\begin{array}{l}
\varepsilon v_{1 t}(t, x)-v_{1 x}(t, x)=0, \varepsilon v_{2 t}(t, x)+v_{2 x}(t, x)=0, \\
v_{1}(t, 0)-v_{2}(t, 0)=2 \varepsilon c Z(t), \\
v_{1}(t, 1)+v_{2}(t, 1)=-d v_{1}(t, 1)+d v_{2}(t, 1), \\
\dot{Z}(t)=a Z(t)+\frac{b}{2 \varepsilon} v_{1}(t, 1)-\frac{b}{2 \varepsilon} v_{2}(t, 1) .
\end{array}\right.
$$

System (22) is a system of fast conservation laws coupled with an ODE. However, due to the presence of $\varepsilon$ in the denominator of the last line of (22) and of the presence of $\varepsilon$ in the second line, it differs from the class of coupled systems of conservation laws and ODE studied in [19]. Therefore the results in [19] do not apply to (22), as claimed in the Introduction.

\section{B. A technical lemma and its proof}

In this section, we prove a technical lemma. Roughly speaking this lemma analyses the stability of a general coupled ODE/hyperbolic system with constant coefficients in the internal and boundary couplings. It will be used in the proofs of Theorem 1 and Theorem 2 .

Lemma 1: Let $\varepsilon, A, B, C, D, E, F, G, H, M$ be constant values, and $d_{1}, d_{2}, d_{3}$ be functions in $C([0, \infty))$. Let us consider the system

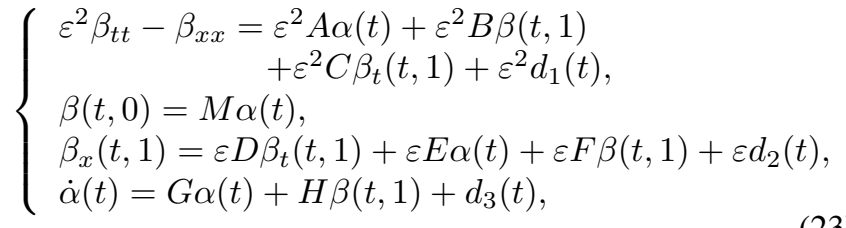

and the Lyapunov function candidate $V(\beta, \alpha)=V_{1}(\beta)+$ $V_{2}(\alpha)$, where $V_{2}(\alpha)=\alpha^{2}$ and

$$
V_{1}(\beta)=\int_{0}^{1} e^{\mu x}\left(\beta_{x}+\varepsilon \beta_{t}\right)^{2} d x+\int_{0}^{1} e^{-\mu x}\left(\beta_{x}-\varepsilon \beta_{t}\right)^{2} d x .
$$


Then, there exists $C_{1}>0$ such that, for any positive values $\kappa_{i}, i=1, \ldots, 7$, we have along all solutions to (23)

$$
\begin{gathered}
\frac{d}{d t} V(\beta, \alpha) \leq\left[-\frac{\mu}{\varepsilon}+e^{\mu}\left(\kappa_{1}+\kappa_{2}+\kappa_{3}+\kappa_{4}\right)+\frac{2 \varepsilon^{2} B^{2} C_{1}}{\kappa_{2}}\right. \\
\left.+3 \varepsilon C_{1} F^{2}\left(e^{\mu}+e^{-\mu}\right)\left(1+\frac{|D|+1}{\kappa_{5}}\right)+6 M^{2} C_{1} \varepsilon H^{2}+\frac{C_{1} H^{2}}{\kappa_{6}}\right] V_{1}(\beta) \\
+\left[2 G+\kappa_{6}+\kappa_{7}+3 M^{2} \frac{H^{2}}{\kappa_{6}}+\frac{2 \varepsilon^{2} A^{2}}{\kappa_{1}}\right. \\
+3 \varepsilon E^{2}\left(e^{\mu}+e^{-\mu}\right)\left(1+\frac{|D|+1}{\kappa_{5}}\right)+6 M^{2} \varepsilon C_{1} G^{2} \\
\left.+\left(\frac{2 \varepsilon^{2} B^{2}}{\kappa_{2}}+3 \varepsilon F^{2}\left(e^{\mu}+e^{-\mu}\right)\left(1+\frac{|D|+1}{\kappa_{5}}\right)+6 M^{2} \varepsilon H^{2}\right) 3 M^{2}\right] \\
\quad \times V_{2}(\alpha) \\
+\frac{2 \varepsilon^{2}}{\kappa_{4}} d_{1}(t)^{2}+3 \varepsilon\left(e^{\mu}+e^{-\mu}\right)\left(1+\frac{|D|+1}{\kappa_{5}}\right) d_{2}(t)^{2} \\
+\left[6 M^{2} \varepsilon+\frac{1}{\kappa_{7}}\right) d_{3}(t)^{2} \\
+D+1)^{2}-\varepsilon e^{-\mu}(D-1)^{2}+3 \varepsilon \kappa_{5}\left(e^{\mu}+e^{-\mu}\right)(|D|+1) \\
\left.+\frac{2 \varepsilon^{2} C^{2}}{\kappa_{3}}\right] \beta_{t}(t, 1)^{2} . \quad(24)
\end{gathered}
$$

Proof: Along the solutions to (23), we compute

$$
\begin{aligned}
\frac{d}{d t} V_{1}(\beta)= & 2 \int_{0}^{1} e^{\mu x}\left(\beta_{x t}+\varepsilon \beta_{t t}\right)\left(\beta_{x}+\varepsilon \beta_{t}\right) d x \\
& +2 \int_{0}^{1} e^{-\mu x}\left(\beta_{x t}-\varepsilon \beta_{t t}\right)\left(\beta_{x}-\varepsilon \beta_{t}\right) d x \\
= & \frac{2}{\varepsilon} \int_{0}^{1} e^{\mu x}\left(\varepsilon \beta_{x t}+\beta_{x x}\right)\left(\beta_{x}+\varepsilon \beta_{t}\right) d x \\
& -\frac{2}{\varepsilon} \int_{0}^{1} e^{-\mu x}\left(-\varepsilon \beta_{x t}+\beta_{x x}\right)\left(\beta_{x}-\varepsilon \beta_{t}\right) d x \\
& +2(\varepsilon A \alpha(t)+\varepsilon B \beta(t, 1) \\
& \left.+\varepsilon C \beta_{t}(t, 1)+\varepsilon d_{1}(t)\right) \int_{0}^{1} e^{\mu x}\left(\beta_{x}+\varepsilon \beta_{t}\right) d x \\
& -2\left(\varepsilon A \alpha(t)+\varepsilon B \beta(t, 1)+\varepsilon C \beta_{t}(t, 1)\right. \\
& \left.+\varepsilon d_{1}(t)\right) \int_{0}^{1} e^{-\mu x}\left(\beta_{x}-\varepsilon \beta_{t}\right) d x
\end{aligned}
$$

and thus, using integrations by parts,

$$
\begin{aligned}
\frac{d}{d t} V_{1}(\beta)= & -\frac{\mu}{\varepsilon} \int_{0}^{1} e^{\mu x}\left(\beta_{x}+\varepsilon \beta_{t}\right)^{2} d x \\
& -\frac{\mu}{\varepsilon} \int_{0}^{1} e^{-\mu x}\left(\beta_{x}-\varepsilon \beta_{t}\right)^{2} d x \\
& +\frac{1}{\varepsilon}\left[e^{\mu x}\left(\beta_{x}+\varepsilon \beta_{t}\right)^{2}-e^{-\mu x}\left(\beta_{x}-\varepsilon \beta_{t}\right)^{2}\right]_{0}^{1} \\
& +2\left(\varepsilon A \alpha(t)+\varepsilon B \beta(t, 1)+\varepsilon C \beta_{t}(t, 1)\right. \\
& \left.+\varepsilon d_{1}(t)\right) \int_{0}^{1} e^{\mu x}\left(\beta_{x}+\varepsilon \beta_{t}\right) d x \\
& -2\left(\varepsilon A \alpha(t)+\varepsilon B \beta(t, 1)+\varepsilon C \beta_{t}(t, 1)\right. \\
& \left.+\varepsilon d_{1}(t)\right) \int_{0}^{1} e^{-\mu x}\left(\beta_{x}-\varepsilon \beta_{t}\right) d x
\end{aligned}
$$

Now using the inequalities $2 e f \leq \frac{e^{2}}{k}+k f^{2},(e+f+g)^{2} \leq$ $3\left(e^{2}+f^{2}+g^{2}\right)$ (for any values $e, f$, and $g$ and any positive value $k$ ), and (23), we get

$$
\begin{aligned}
& \frac{d}{d t} V_{1}(\beta) \leq-\frac{\mu}{\varepsilon} V_{1}(\beta)+\left\{\left[\int_{0}^{1} e^{\mu x}\left(\beta_{x}+\varepsilon \beta_{t}\right) d x\right]^{2}\right. \\
& \left.+\left[\int_{0}^{1} e^{-\mu x}\left(\beta_{x}-\varepsilon \beta_{t}\right) d x\right]^{2}\right\}\left(\kappa_{1}+\kappa_{2}+\kappa_{3}+\kappa_{4}\right) \\
& +\frac{2 \varepsilon^{2} A^{2}}{\kappa_{1}} \alpha(t)^{2}+\frac{2 \varepsilon^{2} B^{2}}{\kappa_{2}} \beta(t, 1)^{2}+\frac{2 \varepsilon^{2} C^{2}}{\kappa_{3}} \beta_{t}(t, 1)^{2} \\
& +\frac{2 \varepsilon^{2}}{\kappa_{4}} d_{1}(t)^{2} \\
& +\frac{e^{\mu}}{\varepsilon}\left[\varepsilon(D+1) \beta_{t}(t, 1)+\varepsilon E \alpha(t)+\varepsilon F \beta(t, 1)+\varepsilon d_{2}(t)\right]^{2} \\
& -\frac{e^{-\mu}}{\varepsilon}\left[\varepsilon(D-1) \beta_{t}(t, 1)+\varepsilon E \alpha(t)+\varepsilon F \beta(t, 1)+\varepsilon d_{2}(t)\right]^{2} \\
& +\frac{2}{\varepsilon} M^{2} \varepsilon^{2}\left(G \alpha(t)+H \beta(t, 1)+d_{3}(t)\right)^{2} \\
& \leq-\frac{\mu}{\varepsilon} V_{1}(\beta)+\left\{e^{\mu} \int_{0}^{1} e^{\mu x}\left(\beta_{x}+\varepsilon \beta_{t}\right)^{2} d x\right. \\
& \left.+\int_{0}^{1} e^{-\mu x}\left(\beta_{x}-\varepsilon \beta_{t}\right)^{2} d x\right\}\left(\kappa_{1}+\kappa_{2}+\kappa_{3}+\kappa_{4}\right) \\
& +\left(\frac{2 \varepsilon^{2} A^{2}}{\kappa_{1}}+6 M^{2} \varepsilon G^{2}\right) \alpha(t)^{2}+\left(\frac{2 \varepsilon^{2} B^{2}}{\kappa_{2}}+6 M^{2} \varepsilon H^{2}\right) \beta(t, 1)^{2} \\
& +\frac{2 \varepsilon^{2} C^{2}}{\kappa_{3}} \beta_{t}(t, 1)^{2}+\frac{2 \varepsilon^{2}}{\kappa_{4}} d_{1}(t)^{2} \\
& +\varepsilon e^{\mu}\left\{(D+1)^{2} \beta_{t}(t, 1)^{2}+\left(E \alpha(t)+F \beta(t, 1)+d_{2}(t)\right)^{2}\right. \\
& \left.+2\left(E \alpha(t)+F \beta(t, 1)+d_{2}(t)\right)(D+1) \beta_{t}(t, 1)\right\} \\
& -\varepsilon e^{-\mu}\left\{(D-1)^{2} \beta_{t}(t, 1)^{2}+\left(E \alpha(t)+F \beta(t, 1)+d_{2}(t)\right)^{2}\right. \\
& \left.+2\left(E \alpha(t)+F \beta(t, 1)+d_{2}(t)\right)(D-1) \beta_{t}(t, 1)\right\}+6 M^{2} \varepsilon d_{3}(t)^{2} \\
& \leq\left[-\frac{\mu}{\varepsilon}+e^{\mu}\left(\kappa_{1}+\kappa_{2}+\kappa_{3}+\kappa_{4}\right)\right] V_{1}(\beta) \\
& +\left[\frac{2 \varepsilon^{2} A^{2}}{\kappa_{1}}+3 \varepsilon E^{2}\left(e^{\mu}+e^{-\mu}\right)\left(1+\frac{|D|+1}{\kappa_{5}}\right)+6 M^{2} \varepsilon G^{2}\right] \alpha(t)^{2} \\
& +\left[\frac{2 \varepsilon^{2} B^{2}}{\kappa_{2}}+3 \varepsilon F^{2}\left(e^{\mu}+e^{-\mu}\right)\left(1+\frac{|D|+1}{\kappa_{5}}\right)+6 M^{2} \varepsilon H^{2}\right] \beta(t, 1)^{2} \\
& +\frac{2 \varepsilon^{2}}{\kappa_{4}} d_{1}(t)^{2}+6 M^{2} \varepsilon d_{3}(t)^{2}+3 \varepsilon\left(e^{\mu}+e^{-\mu}\right)\left(1+\frac{|D|+1}{\kappa_{5}}\right) d_{2}(t)^{2} \\
& +\left[\varepsilon e^{\mu}(D+1)^{2}-\varepsilon e^{-\mu}(D-1)^{2}+3 \varepsilon \kappa_{5}\left(e^{\mu}+e^{-\mu}\right)(|D|+1)\right. \\
& \left.+\frac{2 \varepsilon^{2} C^{2}}{\kappa_{3}}\right] \beta_{t}(t, 1)^{2}
\end{aligned}
$$

for any positive values $\kappa_{i}, i=1, \ldots, 5$. Using first the Agmon inequality (see Appendix A in [15]) and one boundary condition of $\beta$ in (23), it holds

$$
\begin{aligned}
\beta(t, 1)^{2} \leq & \beta(t, 0)^{2}+2\|\beta(t)\|_{L^{2}(0,1)}\left\|\beta_{x}(t)\right\|_{L^{2}(0,1)} \\
\leq & M^{2} \alpha(t)^{2}+\|\beta(t)\|_{L^{2}(0,1)}^{2} \\
& +\left\|\beta_{x}(t)\right\|_{L^{2}(0,1)}^{2} .
\end{aligned}
$$


Moreover, with the Poincaré inequality (see again Appendix A in [15]) it holds, for any positive value $\kappa$ in $(0,1)$,

$$
\begin{aligned}
\|\beta(t)\|_{L^{2}(0,1)}^{2}= & \int_{0}^{1}(\beta(t, x)-\beta(t, 0))^{2} d x \\
& +2 \beta(t, 0) \int_{0}^{1} \beta(t, x) d x-\beta(t, 0)^{2} \\
\leq & \frac{4}{\pi^{2}}\left\|\beta_{x}(t)\right\|_{L^{2}(0,1)}^{2}+\frac{1}{\kappa} \beta(t, 0)^{2} \\
& +\kappa\|\beta(t)\|_{L^{2}(0,1)}^{2}-\beta(t, 0)^{2} \\
\leq & \frac{4}{\pi^{2}}\left\|\beta_{x}(t)\right\|_{L^{2}(0,1)}^{2}+\left(\frac{1}{\kappa}-1\right) \beta(t, 0)^{2} \\
& +\kappa\|\beta(t)\|_{L^{2}(0,1)}^{2}
\end{aligned}
$$

Thus letting $\kappa=1 / 2$ and one boundary condition of $\beta$ in (23), it is deduced

$$
\|\beta(t)\|_{L^{2}(0,1)}^{2} \leq \frac{8}{\pi^{2}}\left\|\beta_{x}(t)\right\|_{L^{2}(0,1)}^{2}+2 M^{2} \alpha(t)^{2} .
$$

It follows with (25) that

$$
|\beta(t, 1)|^{2} \leq\left(\frac{8}{\pi^{2}}+1\right)\left\|\beta_{x}(t)\right\|_{L^{2}(0,1)}^{2}+3 M^{2} \alpha(t)^{2} .
$$

In order to compare $\left\|\beta_{x}(t)\right\|_{L^{2}(0,1)}^{2}$ with $V_{1}(\beta)$ we notice that

$$
\begin{gathered}
V_{1}(\beta)=\int_{0}^{1} e^{\mu x}\left(\beta_{x}+\varepsilon \beta_{t}\right)^{2} d x+\int_{0}^{1} e^{-\mu x}\left(\beta_{x}-\varepsilon \beta_{t}\right)^{2} d x \\
\geq \int_{0}^{1} e^{-\mu x}\left(\beta_{x}+\varepsilon \beta_{t}\right)^{2} d x+\int_{0}^{1} e^{-\mu x}\left(\beta_{x}-\varepsilon \beta_{t}\right)^{2} d x \\
\geq 2 \int_{0}^{1} e^{-\mu x}\left(\beta_{x}^{2}+\varepsilon^{2} \beta_{t}^{2}\right) d x \geq 2 e^{-\mu} \int_{0}^{1} \beta_{x}^{2} d x
\end{gathered}
$$

Thus, we obtain

$$
|\beta(t, 1)|^{2} \leq C_{1} V_{1}(\beta)+3 M^{2} \alpha(t)^{2},
$$

with $C_{1}=\frac{e^{\mu}}{2}\left(\frac{8}{\pi^{2}}+1\right)$. Therefore, we get

$$
\begin{array}{r}
\quad \frac{d}{d t} V_{1}(\beta) \leq\left[-\frac{\mu}{\varepsilon}+e^{\mu}\left(\kappa_{1}+\kappa_{2}+\kappa_{3}+\kappa_{4}\right)+\frac{2 \varepsilon^{2} B^{2} C_{1}}{\kappa_{2}}\right. \\
\left.+3 \varepsilon C_{1} F^{2}\left(e^{\mu}+e^{-\mu}\right)\left(1+\frac{|D|+1}{\kappa_{5}}\right)+6 M^{2} C_{1} \varepsilon H^{2}\right] V_{1}(\beta) \\
+\left[\frac{2 \varepsilon^{2} A^{2}}{\kappa_{1}}+3 \varepsilon E^{2}\left(e^{\mu}+e^{-\mu}\right)\left(1+\frac{|D|+1}{\kappa_{5}}\right)+6 M^{2} C_{1} \varepsilon G^{2}\right. \\
\left.+\left(\frac{2 \varepsilon^{2} B^{2}}{\kappa_{2}}+3 \varepsilon F^{2}\left(e^{\mu}+e^{-\mu}\right)\left(1+\frac{|D|+1}{\kappa_{5}}\right)+6 M^{2} \varepsilon H^{2}\right) 3 M^{2}\right] \alpha \\
+\frac{2 \varepsilon^{2}}{\kappa_{4}} d_{1}(t)^{2}+3 \varepsilon\left(e^{\mu}+e^{-\mu}\right)\left(1+\frac{|D|+1}{\kappa_{5}}\right) d_{2}(t)^{2}+6 M^{2} \varepsilon d_{3}(t)^{2} \\
+\left[\varepsilon e^{\mu}(D+1)^{2}-\varepsilon e^{-\mu}(D-1)^{2}+3 \varepsilon \kappa_{5}\left(e^{\mu}+e^{-\mu}\right)(|D|+1)\right. \\
\left.+\frac{2 \varepsilon^{2} C^{2}}{\kappa_{3}}\right] \beta_{t}(t, 1)^{2} .
\end{array}
$$

Moreover, using the fourth line of (23), inequality (26) and $2 f g \leq \frac{f^{2}}{k}+k g^{2}$ (for any values $f$ and $g$ and any positive value $k$ ), we have

$$
\begin{aligned}
\frac{d}{d t} V_{2}(\alpha)= & 2 \alpha(t)\left(G \alpha(t)+H \beta(t, 1)+d_{3}(t)\right) \\
= & 2 G V_{2}(\alpha)+2 H \alpha(t) \beta(t, 1)+2 \alpha(t) d_{3}(t) \\
\leq & 2 G V_{2}(\alpha)+\frac{H^{2}}{\kappa_{6}} \beta(t, 1)^{2}+\kappa_{6} \alpha(t)^{2}+\kappa_{7} \alpha(t)^{2} \\
& +\frac{1}{\kappa_{7}} d_{3}(t)^{2} \\
\leq & 2 G V_{2}(\alpha)+\frac{H^{2}}{\kappa_{6}}\left(C_{1} V_{1}(\beta)+3 M^{2} \alpha(t)^{2}\right) \\
& +\kappa_{6} \alpha(t)^{2}+\kappa_{7} \alpha(t)^{2}+\frac{1}{\kappa_{3}} d_{3}(t)^{2} \\
\leq & \left(2 G+\kappa_{6}+\kappa_{7}+3 M^{2} \frac{H^{2}}{\kappa_{6}}\right) V_{2}(\alpha) \\
& +\frac{C_{1} H^{2}}{\kappa_{6}} V_{1}(\beta)+\frac{1}{\kappa_{7}} d_{3}(t)^{2}
\end{aligned}
$$

for any positive values $\kappa_{6}$ and $\kappa_{7}$. Combining the previous inequality with (27) we readily obtain (24). This concludes the proof of Lemma 1.

\section{REFERENCES}

[1] M. Barreau, F. Gouaisbaut, A. Seuret, and R. Sipahi. Input/output stability of a damped string equation coupled with ordinary differential system. International Journal of Robust and Nonlinear Control, 28(18):6053-6069, 2018.

[2] M. Barreau, A. Seuret, F. Gouaisbaut, and L. Baudouin. Lyapunov stability analysis of a string equation coupled with an ordinary differential system. IEEE Trans. Automat. Control, 63(11):3850-3857, 2018.

[3] G. Bastin and J.-M. Coron. Stability and Boundary Stabilization of 1-D Hyperbolic Systems, volume 88 of Progress in Nonlinear Differential Equations and Their Applications. Springer, 2016.

[4] V. F. Butuzov and I. Smurov. Initial-boundary value problem for a singularly perturbed parabolic equation in case of exchange of stability. J. Math. Anal. Appl., 234(1):183-192, 1999.

[5] E. Cerpa and C. Prieur. Effect of time scales on stability of coupled systems involving the wave equation. In 56th IEEE Conference on Decision and Control (CDC), pages 1236-1241, Melbourne, Australia, 2017.

[6] E. Cerpa and C. Prieur. Matlab simulation codes. http: //www.gipsa-lab.grenoble-inp.fr/ christophe. prieur/Codes/wave_tikhonov.zip, 2019.

[7] J.-M. Coron, B. d'Andréa Novel, and G. Bastin. A strict Lyapunov function for boundary control of hyperbolic systems of conservation laws. IEEE Trans. Automat. Control, 52(1):2-11, 2007.

[8] J. Daafouz, M. Tucsnak, and J. Valein. Nonlinear control of a coupled PDE/ODE system modeling a switched power converter with a transmission line. Systems Control Lett., 70:92-99, 2014.

[9] A. Diagne, G. Bastin, and J.-M. Coron. Lyapunov exponential stability of 1-D linear hyperbolic systems of balance laws. Automatica J. IFAC, 48(1):109-114, 2012

[10] E. Fridman. Effects of small delays on stability of singularly perturbed systems. Automatica J. IFAC, 38(5):897-902, 2002.

[11] V.Y. Glizer, E. Fridman, and Y. Feigin. A novel approach to exact slow-fast decomposition of linear singularly perturbed systems with small delays. SIAM J. Control Optim., 55(1):236-274, 2017

[12] L.T. Grujic. Uniform asymptotic stability of nonlinear singularly perturbed and large scale systems. Internat. J. Control, 33(3):481$504,1981$.

[13] J. Hudson and P.K. Sweby. Formulations for numerically approximating hyperbolic systems governing sediment transport. J. Sci. Comput., 19:225-252, 2003

[14] P. Kokotović, H.K. Khalil, and J. O'Reilly. Singular pertrubation methods in control: analysis and design. Academic Press, 1986.

$[15]$ M. Krstic. Delay compensation for nonlinear, adaptive, and PDE systems. Springer, 2009.

[16] C. Prieur and F. Mazenc. ISS-Lyapunov functions for time-varying hyperbolic systems of balance laws. Math. Control Signals Systems, 24(1):111-134, 2012

[17] L.F. Shampine. Two-step Lax-Friedrichs method. Appl. Math. Lett., 18:1134-1136, 2005.

[18] A. Smyshlyaev, E. Cerpa, and M. Krstic. Boundary stabilization of a 1-D wave equation with in-domain antidamping. SIAM J. Control Optim., 48(6):4014-4031, 2010.

[19] Y. Tang and G. Mazanti. Stability analysis of coupled linear ODEhyperbolic PDE systems with two time scales. Automatica J. IFAC, 85:386-396, 2017.

[20] Y. Tang, C. Prieur, and A. Girard. Tikhonov theorem for linear hyperbolic systems. Automatica J. IFAC, 57:1-10, 2015.

[21] Y. Tang, C. Prieur, and A. Girard. Singular perturbation approximation of linear hyperbolic systems of balance laws. IEEE Trans. Automat. Control, 61(10):3031-3037, 2016.

[22] E. Witrant, K. Johansson, and the HynX team. Air flow modelling in deep wells: application to mining ventilation. In IEEE Conference on Automation Science and Engineering, pages 845-850, Arlington, VA, 2008.

[23] Z. Zhou and S. Tang. Boundary stabilization of a coupled wave-ODE system with internal anti-damping. Internat. J. Control, 85(11):16831693, 2012. 